\title{
Glucose-6-phosphate dehydrogenase in rat lung alveolar epithelial cells. An ultrastructural enzyme-cytochemical study
}

\author{
S. Matsubara ${ }^{1,3}$, T. Kato ${ }^{2}$, K. Oshikawa ${ }^{2}$, T. Yamada', T. Takayama ${ }^{1}$, T. Koike ${ }^{1}$, T. Watanabe ${ }^{1}$, \\ A. Izumi ${ }^{1}$, and I. Sato
}

${ }^{1}$ Department of Obstetrics and Gynecology, and ${ }^{2}$ Pulmonary Medicine, Jichi Medical School, Minamikawachimachi 3311-1, Kawachi-gun, Tochigi 329-0498, Japan

Accepted: 2/04/02

Key words: glucose-6-phosphate dehydrogenase, lung alveolar epithelial cells, enzyme-cytochemistry

\section{SUMMARY}

Glucose-6-phosphate dehydrogenase (G6PD) is the key enzyme of the pentose phosphate pathway in carbohydrate metabolism, and it plays an important role in cell proliferation and antioxidant regulation within cells in various organs. Although marked cell proliferation and oxidant/antioxidant metabolism occur in lung alveolar epithelial cells, definite data has been lacking as to whether cytochemically detectable G6PD is present in alveolar epithelial cells. The distribution pattern of G6PD within these cells, if it is present, is also unknown. The purpose of the present study was to investigate the subcellular localization of G6PD in alveolar cells in the rat lung using a newly-developed enzyme-cytochemistry (copper-ferrocyanide) method. Type I cells and stromal endothelia and fibroblasts showed no activities. Electron-dense precipitates indicating G6PD activity were clearly visible in the cytoplasm and on the cytosolic side of the endoplasmic reticulum of type II alveolar epithelial cells. The cytochemical controls ensured specific detection of enzyme activity. This enzyme may play a role in airway defense by delivering substances for cell proliferation and antioxidant forces, thus maintaining the airway architecture.

\section{INTRODUCTION}

The enzyme glucose-6-phosphate dehydrogenase (G6PD; E.C. 1.1.1.49) plays pivotal roles in cell biology in various organs. G6PD catalyzes the conversion of glucose-6-phosphate to 6-phosphogluconate, with the concomitant production of ribose-5-phosphate and NADPH. Ribose-5-phosphate is used for DNA or RNA synthesis in cell proliferation, and NADPH is utilized as a coenzyme for various enzymes that generate reduced glutathione, a potent antioxidant within cells. G6PD, therefore, is considered to play important roles in cell biology in DNA or RNA synthesis and as an antioxidant supplier (Tian et al., 1998).

The lung, especially alveolar epithelial cells, has direct contact with oxygen, and thus this tissue is likely to receive oxygen- or oxidant-induced tissue damage (Fraser et al., 1999). At the same time, alveolar epithelial cells, especially type II epithelial cells, possess high proliferative capacity (Fraser et al., 1999). Considering that G6PD supplies cells with ribose-5-phosphate and NADPH, which are essential substances for cell proliferation and antioxidation, respectively, G6PD in alveolar epithelial cells may play important roles in lung biology. 
Biochemical evidence indicated that alveolar type II epithelial cells possessed high G6PD enzyme activity compared to whole lung tissue homogenates (Forman and Fisher 1981; Freeman et al., 1986). Alhough a previous light microscopic study also suggested the presence of G6PD in rat type II alveolar cells (Negi and Stephens 1977), definite morphological evidence for the presence of G6PD within alveolar cells has not been reported. Is cytochemically detectable G6PD present in alveolar epithelial cells? If it is present, what localization pattern does this enzyme exhibit? Recently, we developed a G6PD enzyme-cytochemistry method using copper ferrocyanide as the capturing agent (copper-ferrocyanide method), which enabled us to determine the fine subcellular localization sites of G6PD within cells, and we found that the G6PD enzyme distribution pattern was quite characteristic (Ishibashi et al., 1999). We performed the present study to investigate the subcellular localization of G6PD in rat alveolar epithelial cells, using the newly developed G6PD enzyme-cytochemistry method.

\section{MATERIALS AND METHODS}

Five 7-week old male Sprague-Dawley rats (200$220 \mathrm{~g}$ ) were used for this study. They were humanely cared for and maintained on ordinary laboratory chow and water ad libitum. Under pentobarbital anesthesia, lungs were perfused with $2.0 \%$ glutaraldehyde solution $(0.1 \mathrm{~mol} / \mathrm{L}$ cacodylate buffer (pH 7.4) with $2 \%$ sucrose) via the trachea for 10 seconds. Lung tissues were then cut into small strips and fixed in a $2.0 \%$ glutaraldehyde solution $(0.1 \mathrm{~mol} / \mathrm{L}$ cacodylate buffer $(\mathrm{pH} 7.4)$ with $10 \%$ sucrose) for 5 mins at $4{ }^{\circ} \mathrm{C}$. After washing in a cacodylate buffer $(0.1 \mathrm{~mol} / \mathrm{L}, \mathrm{pH} 7.4)$ for $3 \mathrm{hrs}$, the samples were cut into $40 \mu \mathrm{m}$-sections with either a DTK-300 Microslicer (DSK Co., Kyoto, Japan) or a freezing microtome (MB201, Komatsu Electronics, Inc., Japan). Cytochemical detection of G6PD was performed as previously described (Ishibashi et al., 1999). In brief, sections were incubated in a reaction medium for 60 mins at $37^{\circ} \mathrm{C}$ in the dark with constant agitation. The reaction medium consisted of $10.5 \mathrm{mmol} / \mathrm{L}$ G6P (disodium salt; the substrate), 70 $\mathrm{mmol} / \mathrm{L}$ phosphate buffer $(\mathrm{pH} 7.2), 1.3 \mathrm{mmol} / \mathrm{L}$ NADP, $10 \mathrm{mmol} / \mathrm{L}$ sodium citrate, $1.5 \mathrm{mmol} / \mathrm{L} \mathrm{cop}-$ per sulfate, $0.5 \mathrm{mmol} / \mathrm{L}$ potassium ferricyanide, 1.0 $\mathrm{mmol} / \mathrm{L}$ phenazine methosulfate, and $267 \mathrm{mmol} / \mathrm{L}$ sucrose. Sections were postfixed in $1.0 \%$ buffered osmium tetroxide for 60 mins at $4^{\circ} \mathrm{C}$, dehydrated, and embedded in epoxy resin, Quetol 812 (Nisshin EM Co., Tokyo, Japan). Ultrathin sections were prepared with an LKB Ultrotome III and observed under a Hitachi H-7000 transmission electron microscope. G6PD activity was visible as electrondense deposits of copper ferrocyanide via the following mechanism: G6P (the substrate) oxidation by G6PD generates free electrons $(\mathrm{H}+)$, which are transferred to NADP and then to phenazine methosulfate (an exogenous electron carrier), and finally trapped by ferricyanide, leading to electron-dense copper-ferrocyanide formation at the exact site of the G6PD enzyme molecules (Ishibashi et al., 1999). To ensure the specific detection of G6PD activity, cytochemical control experiments were performed as follows: (1) sections were incubated in a medium (a) lacking the substrate (G6P), (b) devoid of NADP, or (c) containing $2.0 \mathrm{mmol} / \mathrm{L} \mathrm{p}$ chloromercuribenzoate (an inhibitor of G6PD activity); and (2) sections were heated at $100^{\circ} \mathrm{C}$ for $10 \mathrm{~min}$, then incubated in the complete reaction medium. As a positive tissue control, cytochemical detection of G6PD was performed in the rat liver as previously described (Ishibashi et al., 1999; Matsubara et al., 2001a).

\section{RESULTS}

The alveolar epithelium consists of two different cells; type I and type II cells. Type I cells have flattened cytoplasm, covering approximately $96 \%$ of the alveolar surface (Hirai et al., 1984). Type II cells are cuboidal cells and have some microvilli toward the airway. Type II cells synthesize and secrete alveolar surfactant and possess lamellar body within their cytoplasm. These morphological features enabled us to recognize them as type II alveolar cells. Beneath the epithelium, there are alveolar stroma, in which there are capillary endothelial cells and fibroblasts.

In the rat liver (positive tissue control), very dense precipitates of G6PD were visible in the cytoplasm and on the cytosolic side of the endoplasmic reticulum (ER) (Figs. 1A and 1B), agreeing well with the previous observations by our group (Ishibashi et al., 1999; Matsubara et al., 2001a; b). In the rat 
lung, precipitates indicating G6PD activity were clearly visible in the cytoplasm and on the cytosolic side of ER membranes of type II alveolar epithelial cells (Figs. 2A, 2B and 2D). The intensities and distribution patterns of type II cell G6PD labeling showed no regional/zonal differences within the terminal airways observed. Type I alveolar epithelial cells did not show any G6PD labeling (Fig. 2C). Alveolar mesencymal cells (endothelial cells and fibroblasts) did not exhibit G6PD labeling either (data not shown). Deposits were not visible in cytochemical negative control experiments (Fig. 2E). These findings did not differ among subjects studied.

\section{DISCUSSION}

In the present study, deposits indicative of G6PD were observed both in the cytoplasm and on the cytosolic side of ER of rat type II lung alveolar epithelial cells. Type I cells were devoid of activity. The following observations coincided well with the present findings: 1) Previous light microscopic nitroblue tetrazolium-histochemistry showed G6PD labeling in rat type II alveolar cell cytoplasm (Negi and Stephens 1977); 2) biochemical data also indicated that rat type II cells contained much higher levels of G6PD activity compared to whole lung tissue homogenates (Forman and Fisher 1981; Freeman et al., 1986); 3) the subcellular localization pattern of enzyme-cytochemically detectable G6PD in tissues other than the lung (i.e. the rat liver, the human placenta) was identical to that in the rat type II cells observed here (Ishibashi et al., 1999; Matsubara et al., 2001a; b), and; 4) biochemical (Ozols, 1993) and immuno-electron microscopic (Ninfali et al., 2000) studies demonstrated that G6PD resided both in cytoplasm and in close association with ER in rabbit hepatocytes and rat central nervous system neurons, respectively. The present positive/negative cytochemical control experiments confirmed the specific detection of G6PD demonstrated here. Thus, we conclude that enzyme-cytochemically detectable G6PD was localized both in the cytoplasm and in close association with the ER of rat type II alveolar epithelial cells.

Regarding the biological significance of this G6PD in type II alveolar cells, two possible roles can be considered. First, the G6PD of type II cells may play a role as a potent antioxidant force in the lung. Alveolar epithelial cells always have direct contact with high concentrations of oxygen, and thus are vulnerable to oxygen- or oxidant-induced tissue injuries (Warshaw et al., 1985; Rahman and MacNee 2000). G6PD supplies cells with $\mathrm{NADPH}$, which itself is an antioxidant and also serves as a coenzyme of various glutathione-related reductases, which generate reduced glutathione, one of the most potent antioxidants within alveolar cells (Rahman and MacNee 2000). In the rat, exposure to high concentrations of oxygen induced increased activity in type II alveolar cell G6PD (Warshaw et al., 1985; Crouch et al., 1988). Oxidative stress also affected the G6PD activity of both human type II alveolar cell lines and isolated rat type II cells (Rahman et al 1995). The G6PD of type II cells may play a role in host airway defense against oxygen toxicity by its antioxidant generating capacity in normal lung physiology.

Second, G6PD in type II cells may contribute to alveolar cell proliferation, and thus to maintenance of the airway tissue architecture, by supplying ribose-5-phosphate to DNA or RNA synthesis within these cells. Previously, we demonstrated subcellular localization of various enzymes in human placental trophoblasts (Matsubara et al., 2001c), including G6PD (Matsubara et al., 2001a). G6PD activity was marked in cytotrophoblasts compared to syncytiotrophoblasts (Matsubara et al., 2001a). In human placental villi, when surface syncytiotrophoblasts die, the underlying cytotrophoblasts proliferate and fuse to the dying syncytium, becoming new syncytiotrophoblasts. Cytotrophoblasts are thus considered reserve cells and have high proliferative capacity (Castellucci and Kaufmann 2000). We concluded that cytotrophoblastic high G6PD activity may be associated with the proliferative capacity of cytotrophoblasts as reserve cells, contributing to the maintenance of villous tree architecture (Matsubara et al., 2001a). This suggested function of placental cytotrophoblastic G6PD may hold true for G6PD of type II alveolar epithelial cells. Type I cells are susceptible to various external toxins, and when they die, type II cells proliferate and differentiate into type I cells, thus restoring the normal alveolar epithelia (Hirai et al., 1984; Fraser et al., 1999; Limper and Rosenow 2000). Therefore, although type II cells have a specific function such as surfactant production, they are considered to be reserve 

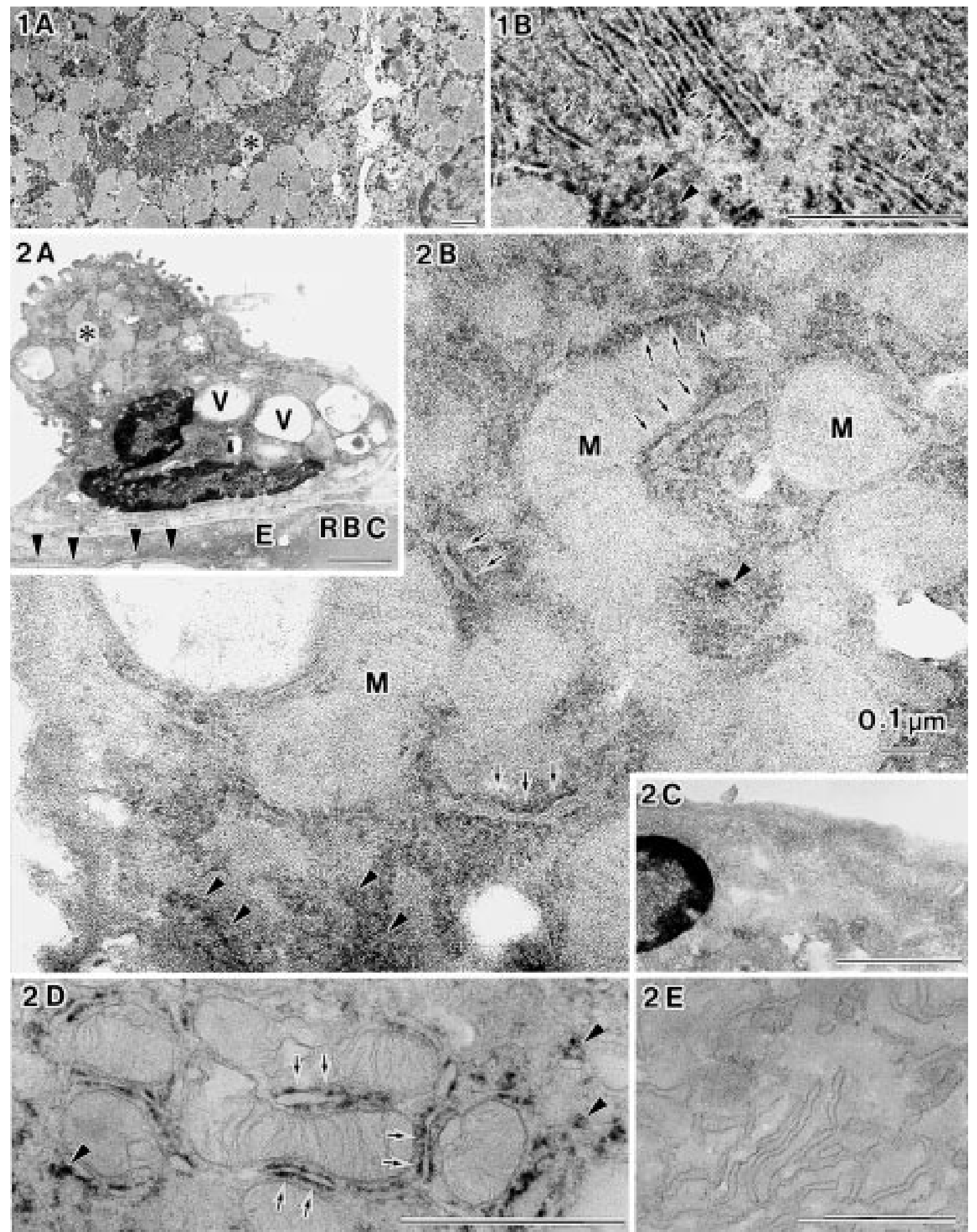
cells for type I cells, having higher proliferative capacity than the type I cells. Since type II alveolar cells are reserve cells for type I cells in the repair of airway damage, and since G6PD in placental reserve cells (cytotrophoblasts) may contribute to cell proliferation, it is easy to hypothesize that, in the lung, type II cell G6PD may also play a role in cell proliferation and in the maintenance of airway architecture. We did not produce direct evidence for these two possible functions of type II cell G6PD, thus, they remain hypothetical, though reasonable.

It is now clear that the cytoplasm and the cytosolic area of ER of alveolar type II cells are sites of G6PD activity. An imbalance favoring oxidant over antioxidant forces is considered to play a pivotal role in the pathogenesis and progression of various lung diseases (Fraser et al., 1999; Rahman and MacNee 2000; Limper and Rosenow 2000). Furthermore, the proliferative state of type II cells affects vulnerability to drug-induced pulmonary injury (Limper and Rosenow 2000). The distribution and intensities of type II cell G6PD, one of the essential intracellular regulators of cell proliferation capacity and the oxidant/antioxidant balance, may be expected to change in pathological lung conditions. Future studies on alveolar cell G6PD may shed light on this important topic.

\section{REFERENCES}

Castellucci M., and Kaufmann P.: Basic structure of the villous trees. In Pathology of the human placenta (Eds. Benirschke K., and Kaufmann P.) Springer-Verlag, New York, pp. 57-115, 2000.

Crouch L.S., Prough R.A., Kennedy K.A., Snyder J.B., and Warshaw J.B.: Rat lung antioxidant enzyme activities and their specific proteins during hyperoxia. J. Appl. Physiol. 65, 797-804, 1988.

Forman H.J., and Fisher A.B.: Antioxidant enzymes of rat granular pneumocytes. Lab. Invest. 45, 1-6, 1981.

Fraser R.S., Colman N., Muller N.L., and Pare P.D.: Inhaled toxic gases, fumes, and aerosols. In Fraser and Pare's diagnosis of diseases of the chest (Eds. Fraser R.S., Colman N., Muller N.L., and Pare P.D.) WB Saunders, Philadelphia, pp. 2519-2535, 1999.

Freeman B.A., Mason R.J., Williams M.C., and Crapo J.D.: Antioxidant enzyme activity in alveolar type II cells after exposure of rats to hyperoxia. Exp. Lung Res. 10, 203-222, 1986.

Hirai K., Uyeda T., and Ogawa K.: Electron-cytochemical studies on the differentiation of mouse lung alveolar epithelial cells with special reference to changes in mitochondria. Acta Histochem. Cytochem. 17, 197-211, 1984.

Ishibashi T., Takizawa T., Iwasaki H., Saito T., Matsubara S., Nakazawa E., and Kanazawa K.: Glucose-6-phosphate dehydrogenase cytochemistry using copper ferrocyanide and its application to rapidly frozen cells. Histochem. Cell Biol. 112, 221-32, 1999.

Limper A.H., and Rosenow III E.C.: Drug-induced pulmonary disease. In Textbook of respiratory medicine. (Eds. Limper A.H., and Rosenow III E.C.) Saunders, Philadelphia, pp. 1971-1994, 2000.

Matsubara S., Takizawa T., Suzuki T., Minakami H., and Sato I.: Glucose-6-phosphate dehydrogenase is present in normal and preeclamptic placental trophoblasts-ultrastructural enzymehistochemical evidence. Placenta 22, 90-95, 2001a.

Matsubara S., Takizawa T., Takayama T., Iwasaki R., Minakami H., and Sato I.: Cytochemically detectable glucose-6-phosphate dehydrogenase is present in human fetal membrane chorion laeve trophoblasts. Placenta 22, 613-616, $2001 \mathrm{~b}$.

Matsubara S., Takayama T., Iwasaki R., Izumi A., Watanabe T., and Sato I.: Chorion laeve trophoblasts of preeclamptic fetal membranes: histochemically detectable enzyme activities do not change at a subcellular level. Eur. J. Histochem. 45, 211217, 2001c.

Fig. 1 - Rat liver cell G6PD activity (positive tissue control). The area indicated by an asterisk in (A) is shown in (B) at a higher magnification. Electron-dense precipitates indicative of G6PD activity were visible on the cytosolic side of endoplasmic reticulum (ER) (arrows) and in the cytoplasm (arrowheads). Bars indicate $1 \mu \mathrm{m}$

Fig. 2 - G6PD activity in the rat lung alveolar epithelium and cytochemical negative control. A. Type II alveolar epithelial cell. Though G6PD labeling is not clearly seen at this low magnification, this type II cell possesses electron-dense (darker) cytoplasm, possibly due to cytoplasmic G6PD deposits, which are identified in (B) at a higher magnification. Cytoplasmic vacuoles (V) are visible, which may be lamellar bodies losing their contents, possibly due to freezing and thawing during 40- $\mu \mathrm{m}$ sectioning for cytochemistry. E; Endothelial cell, RBC; red blood cell in the capillary. Arrowheads indicate flattened cytoplasm of type I cell covering the alveolar surface. The area marked by an asterisk is shown in (B) at a higher magnification. B. G6PD activity in type II alveolar epithelial cells. Deposits indicative of G6PD activity are clearly visible on the cytosolic side of ER (arrows) and in the cytoplasm (arrowheads). Mitochondria (M) are evident. C. Type I alveolar epithelial cell. G6PD labeling is not observed. D. G6PD labeling in cytoplasm of another Type II cell. Unstained specimen. Omission of counterstaining made the deposits more clearly highlighted on the cytosolic side of ER (arrows) and in the cytoplasm (arrowheads). E. Cytochemical negative control. Unstained specimen. Omission of NADP from the reaction medium completely abolished the cytochemically-detectable G6PD labeling in type II cell cytoplasm. Bars indicate $1 \mu \mathrm{m}$ except for $2 \mathrm{~B}$. 
Negi D.S., and Stephens R.J.: An improved method for the histochemical localization of glucose-6-phosphate dehydrogenase in animal and plant tissues. J. Histochem. Cytochem. 25, 149-154, 1977.

Ninfali P., Biagiotti E., Guidi L., Malatesta M., Gazzanelli G., and Grande P.D.: Cytochemical and immunocytochemical methods for electron microscopic detection of glucose-6phosphate dehydrogenase in brain areas. Brain Res. Protoc. 5, 115-120, 2000.

Rahman I., and MacNee W.: Oxidative stress and regulation of glutathione in lung inflammation. Eur. Respir. J. 16, 534$554,2000$.
Rahman I., Li X.Y., Donaldson K., Harrison D.J., and MacNee W.: Glutathione homeostasis in alveolar epithelial cells in vitro and lung in vivo under oxidative stress. Am. J. Physiol. 269, L285-292, 1995.

Tian W.N., Braunstein L.D., Pang J., Stuhlmeier K.M., Xi Q.C., Tian X., and Stanton R.C.: Importance of glucose-6phosphate dehydrogenase activity for cell growth. J. Biol. Chem. 273, 10609-10617, 1998.

Warshaw J.B., Wilson C.W., Saito K., and Prough R.A.: The responses of glutathione and antioxidant enzymes to hyperoxia in developing lung. Pediatr. Res. 19, 819-823, 1985. 